\title{
Condições de saúde e nível de atividade física em idosos participantes e não participantes de grupos de convivência de Florianópolis
}

\author{
Health status and physical activity levels among the elderly \\ who are participants and non-participants \\ in social welfare groups in Florianópolis
}

Tânia Rosane Bertoldo Benedetti ${ }^{1}$

Giovana Zarpellon Mazo ${ }^{1}$

Lucélia Justino Borges ${ }^{1}$

${ }^{1}$ Departamento de Educação Física, Centro de Desportos, Universidade Federal de Santa Catarina. Campus Universitário $\mathrm{Cx}$ postal 476, Trindade. 88010-970 Florianopolis SC.

benedetti@cds.ufsc.br

\begin{abstract}
This study sought to verify the association between health status and physical activity levels among the elderly who are participants and non-participants in social welfare groups in Florianópolis in the State of Santa Catarina, Brazil. The sample included 1,062 elderly people (625 women), mean age 71.9 ( \pm 7.6$)$. The variables analyzed were gender, age, schooling, marital status, physical activity levels (International Physical Activity Questionnaire) and physical health status information (Brazil Elderly Schedule Questionnaire). Data were analyzed by Chi-square test. The results revealed that $60.6 \%$ were classified as physically active (total physical activity level) and $74 \%$ of the elderly reported illness. Illness status was more prevalent among social welfare group participants than non-participants. However, a better positive perception of physical health status was observed among social groups participants. For women, participation in social welfare groups was associated with a positive perception of physical health status $(p<0.001)$ and with illness $(p=0.005)$. The conclusion was that participation in social welfare groups contributes to a better perception of physical health status, as well as for the maintenance of adequate physical activity levels.
\end{abstract}

Key words Health status, Physical activity, Social groups, The elderly
Resumo Objetivou-se verificar a associação entre condições de saúde e nível de atividade física em idosos participantes e não participantes de grupos de convivência de Florianópolis, Santa Catarina. A amostra foi constituída de 1.062 idosos (625 mulheres), com média de idade de 71,9 anos $( \pm 7,6)$. Foram analisadas as variáveis sociodemográficas (sexo, idade, escolaridade e estado civil), nível de atividade física (International Physical Activity Questionaire) e saúde física (Questionário Brazil Older Aging Schedule). Os dados foram analisados por meio da estatística descritiva e teste Qui-quadrado. Os resultados demonstraram que $60,6 \%$ dos idosos foram classificados como ativos fisicamente. A presença de doenças foi relatada por $74 \%$ dos idosos, sendo que os participantes de grupos de convivência (GP) apresentaram maior prevalência que o grupo não participante (GNP). Porém, mesmo com maior prevalência de doença, os idosos do GP apresentaram percepção positiva do estado de saúde. Para as mulheres, a participação em grupos de convivência associouse positivamente com percepção do estado de saúde $(p<0,001)$ e com presença de doença $(p=$ $0,005)$. Conclui-se que a participação nos grupos de convivência contribui para a melhor percepção do estado de saúde e para a manutenção de níveis adequados de atividade física.

Palavras-chave Condições de saúde, Atividade física, Idoso 


\section{Introdução}

O envelhecimento populacional acarreta desafios para a sociedade, fato que altera a demanda por políticas públicas na distribuição dos recursos econômicos. O elevado custo na assistência à saúde, a distribuição de renda inadequada, baixo grau de escolaridade, condições precárias de habitação e do ambiente acarretam repercussões sociais que têm impacto na economia do país, e que afetam a qualidade da vida e da saúde de sua população ${ }^{1,2}$.

Analisando o contexto do envelhecimento populacional no Brasil, principalmente a partir da década de 90, surgiram mobilizações de órgãos governamentais e de segmentos da sociedade civil, que resultaram na Política Nacional do Idoso $^{3}$ e, posteriormente, no Estatuto do Idoso ${ }^{4}$. Como implementação dessas leis encontra-se a estratégia do atendimento por meio de centros de convivência, que consistem em atividades que visam o fortalecimento de atividades associativas, produtivas e de promoção da sociabilidade, contribuindo para a autonomia e a prevenção do isolamento social nas pessoas idosas 5 .

A participação dos idosos em grupos de convivência pode afastar a solidão e propiciar amizades $^{6}$; aumento da autoestima e melhorar a integração com familiares, resgatando valores pessoais e sociais ${ }^{7}$; bem como o suporte social que é um fator importante para eles ${ }^{6,8}$. Além dessas características, a participação em grupos de convivência pode favorecer a adoção de um estilo de vida mais ativo, pois nestes são realizadas atividades de lazer, culturais, intelectuais, físicas, manuais, artísticas e de convívio grupal ${ }^{9}$.

Pesquisas que avaliaram o nível de atividade física de idosos participantes de grupos de convivência verificaram que estes são mais ativos fisicamente ${ }^{10,11}$ e que as mulheres idosas são mais ativas que os homens idosos ${ }^{11}$. Foi observado que o baixo nível de atividade física mostrou-se associado às variáveis sociodemográficas (idade, estado civil, etnia, escolaridade), à presença de doença e ao estado de saúde que dificultava a prática de atividade física ${ }^{10}$. Além disso, idosas participantes de grupos de convivência que são fisicamente ativas têm melhor qualidade de vida ${ }^{12}$.

Porém, poucos estudos epidemiológicos avaliaram a associação entre as condições de saúde com o nível de atividade física em idosos participantes e não participantes de grupos de convivência. Dessa forma, torna-se necessário conhecer tal associação, na tentativa de auxiliar a criação de programas adequados à realidade do ido- so, visando à promoção do bem estar e a melhora da qualidade de vida desta população.

Nesse sentido, o objetivo deste estudo foi verificar a associação entre as condições de saúde e o nível de atividade física em idosos participantes e não participantes de grupos de convivência de Florianópolis, Santa Catarina.

\section{Materiais e métodos}

\section{População e Amostra}

Este estudo epidemiológico transversal teve como população os idosos (de ambos os sexos) residentes no município de Florianópolis, Santa Catarina, Brasil. De acordo com o censo de 2010, a população de Florianópolis é de 421.240, sendo que o número de idosos é 48.423 (20.529 homens e 27.894 mulheres). Entretanto, considerando o ano da coleta de dados, foram utilizados os dados do censo de 2000 para o cálculo da amostra. Este município, no ano de 2000, apresentava uma população de idosos de 28.816 (11.979 homens e 16.837 mulheres), o que representava $8,4 \%$ da população total ${ }^{13}$. Destes, aproximadamente $98 \%$ residiam em domicílio urbano ${ }^{13}$.

Para composição da amostra deste estudo foram utilizados dados provenientes de duas pesquisas $^{14,15}$, os quais foram reorganizados para a análise das condições de vida e nível de atividade física, de acordo com a participação dos idosos em grupos de convivência. As amostras foram selecionadas de forma probabilística e estratificadas por sexo e idade ${ }^{15}$, e somente por idade ${ }^{14}$, com cálculos amostrais. As unidades primárias para seleção foram os setores censitários definidos pelo Instituto Brasileiro de Geografia e Estatística ${ }^{15} \mathrm{e}$ os distritos municipais ${ }^{14}$, e como unidades secundárias foram os domicílios ${ }^{15} \mathrm{e}$ os grupos de convivência existentes em Florianópolis no ano de $2002^{14}$. Foram avaliadas 198 idosas participantes de grupos de convivência em Florianópolis ${ }^{14}$ e 864 idosos residentes na comunidade no ano de $2002^{15}$. Noventa e cinco idosos $(10,8 \%)$ da amostra de Benedetti (2004) participavam de grupos de convivência. Assim, foram constituídos dois grupos: participantes (GP) e não participantes (GNP) em grupos de convivência para idosos; totalizando 1.062 idosos, sendo $27,6 \%(n=293)$ no GP, e $72,4 \%(n=769)$ no GNP.

Foram obtidos pareceres favoráveis dos Comitês de Ética em Pesquisas em Seres Humanos da Universidade Federal de Santa Catarina e da Universidade do Estado de Santa Catarina. 


\section{Instrumentos e coleta de dados}

Os dados sociodemográficos (sexo, idade, estado civil e escolaridade) foram avaliados por meio da aplicação de uma ficha diagnóstica e as condições de saúde (percepção do estado de saúde e presença de doença) foram avaliadas pela seção de saúde física do questionário Brazil Older Aging Schedule - $\mathrm{BOAS}^{16}$. O nível de atividade física (total e nos diferentes domínios - trabalho, transporte, atividades domésticas e lazer) foi obtido pelo Questionário Internacional de Atividade Física (IPAQ), versão longa, referente à uma semana normal. Os dados foram interpretados utilizando o critério baseado nas recomendações de atividades físicas que resultam em benefícios para a saúde, classificando como "ativos" os indivíduos que praticavam até 150 minutos por semana ( $\mathrm{min} / \mathrm{sem})$ de atividade física, no mínimo de intensidade moderada ${ }^{17}$.

Os instrumentos foram aplicados em forma de entrevista aos idosos de grupos de convivência (GP), no próprio local onde eram realizadas as atividades do grupo, e para o GNP, nos seus domicílios. A equipe de coleta de dados foi composta por entrevistadores previamente treinados e supervisionados pelas pesquisadoras responsáveis pelo estudo.

\section{Tratamento estatístico}

As variáveis sociodemográficas foram categorizados por sexo (feminino e masculino); idade em estratos etários (60-69; 70-79; 80 e mais anos); estado civil (com companheiro e sem companheiro); escolaridade (nenhuma; até $4^{\text {a }}$ série; fundamental incompleto; ensino médio; superior). As condições de saúde foram categorizadas em presença de doença (sim e não) e percepção do estado de saúde (boa e ruim). O nível de atividade física foi dicotomizado em pouco ativo $(<150 \mathrm{~min} / \mathrm{sem})$ e ativo $(\geq 150 \mathrm{~min} / \mathrm{sem})$.

Foi realizada estatística descritiva (frequência, percentual, média e desvio padrão) e para verificar as associações entre condições de saúde e nível de atividade foi utilizado o Teste Qui-quadrado. O nível de significância adotado foi de $5 \%$. Os dados foram analisados no programas estatísticos SPSS ${ }^{\circledast} 17.0$ e MedCalc.

\section{Resultados}

A idade variou de 60 a 101 anos, sendo a média geral de 71,9 anos $( \pm 7,6)$. A média de idade do GP foi de 72,6 ( $\pm 6,4)$ e do GNP foi de 71,7 ( \pm
$8,0)$. O GP é caracterizado por mulheres que vivem sem companheiro e a faixa etária 70 a 79 anos foi a prevalente. No GNP há maior prevalência de idosos jovens (60-69 anos) e que vivem acompanhados. A baixa instrução foi prevalente em ambos os grupos (Tabela 1).

Em relação ao nível de atividade física total, a maioria dos idosos $(60,6 \%)$ foi classificada como ativos. Quando realizada a análise por domínios e por grupos, foi observado que a maioria do GP e do GNP foi considerada pouco ativa em todos os domínios. Ressalta-se que dentre os ativos fisicamente, as maiores prevalências foram observadas nos domínios das atividades domésticas e de lazer para o GP, e atividade de lazer e transporte para o GNP. Foi evidenciada diferença significante das proporções dos idosos poucos ativos no transporte e nas atividades domésticas entre o GP e o GNP. Para os ativos foi observada diferença significante da proporção das atividades domésticas entre o GP e o GNP (Tabela 2).

Observa-se na Tabela 3, para ambos os grupos, que a maioria dos idosos tem doenças, percepção positiva do seu estado de saúde e são ativos fisicamente. Contudo, foi detectada associação estatística significante entre presença de doença e percepção do estado de saúde com a participação em grupos de convivência, somente para o sexo feminino (Tabela 3 ).

\section{Discussão}

Os resultados do presente estudo evidenciaram associação estatística significante entre condições de saúde e participação em grupos de convivência para as idosas, não sendo detectada associação com o nível de atividade física. Os idosos participantes do GP são mais ativos fisicamente (nível de atividade física total), têm maior número de doenças e melhor percepção do estado de saúde que os idosos do GNP.

As mulheres participam mais dos grupos de convivência que os homens. As melhores condições de vida contribuem para o aumento da expectativa de vida e da longevidade, sendo que as mulheres vivem mais que os homens. Dessa forma, especialmente a longevidade feminina, acarreta um número expressivo de viúvas participando de grupos de convivência ${ }^{18}$. Isso pode ser observado na presente pesquisa, uma vez que do total de mulheres $(n=266)$ inseridas nesses grupos, $59,8 \%$ eram viúvas.

Entre os idosos do GP, ainda é verificada baixa escolaridade (até quarta série do ensino fun- 
Tabela 1. Distribuição das variáveis sociodemográficas, segundo a participação ou não em grupos de convivência para idosos. Florianópolis, Santa Catarina, 2002.

\begin{tabular}{|c|c|c|c|c|c|c|}
\hline & \multicolumn{2}{|c|}{$\begin{array}{l}\text { Participantes } \\
\qquad(\mathbf{n}=293)\end{array}$} & \multicolumn{2}{|c|}{$\begin{array}{l}\text { Não participantes } \\
\quad(n=769)\end{array}$} & \multicolumn{2}{|c|}{$\begin{array}{c}\text { Total } \\
(\mathrm{n}=1062)\end{array}$} \\
\hline & $\mathbf{n}$ & $\%$ & $\mathbf{n}$ & $\%$ & $\mathbf{n}$ & $\%$ \\
\hline \multicolumn{7}{|l|}{ Sexo } \\
\hline Masculino & 27 & 9,2 & 410 & 53,3 & 437 & 41,1 \\
\hline Feminino & 266 & 90,8 & 359 & 46,7 & 625 & 58,9 \\
\hline \multicolumn{7}{|l|}{ Idade (anos) } \\
\hline $60-69$ & 110 & 37,5 & 348 & 45,3 & 458 & 43,1 \\
\hline $70-79$ & 138 & 47,1 & 283 & 36,8 & 421 & 39,6 \\
\hline 80 ou mais & 45 & 15,4 & 138 & 17,9 & 183 & 17,2 \\
\hline \multicolumn{7}{|l|}{ Estado civil } \\
\hline Com companheiro & 103 & 35,2 & 486 & 63,2 & 589 & 55,5 \\
\hline Sem companheiro & 190 & 64,8 & 283 & 36,8 & 473 & 44,5 \\
\hline \multicolumn{7}{|l|}{ Escolaridade $^{*}$} \\
\hline Nenhuma & 35 & 12,5 & 47 & 7,1 & 82 & 8,7 \\
\hline Até $4^{\text {a }}$ série & 177 & 63,2 & 328 & 49,6 & 505 & 53,7 \\
\hline Fundamental incompleto & 27 & 9,6 & 89 & 13,5 & 116 & 12,3 \\
\hline Ensino médio & 31 & 11,1 & 101 & 15,3 & 132 & 14,0 \\
\hline Superior & 10 & 3,6 & 96 & 14,5 & 106 & 11,3 \\
\hline
\end{tabular}

${ }^{*}$ Participantes $(\mathrm{n}=280)$ e não participantes $(\mathrm{n}=661)$.

Tabela 2. Comparação dos níveis de atividade física total e por domínios, segundo a participação ou não em grupos de convivência para idosos. Florianópolis, Santa Catarina, 2002.

\begin{tabular}{|c|c|c|c|c|c|c|c|c|c|c|}
\hline \multirow{3}{*}{$\begin{array}{l}\text { Variáveis } \\
\text { Atividade Física }\end{array}$} & \multicolumn{4}{|c|}{$\begin{array}{l}\text { Participantes de grupo de } \\
\text { convivência }(\mathrm{n}=293)\end{array}$} & \multicolumn{4}{|c|}{$\begin{array}{l}\text { Não participantes de grupos } \\
\text { de convivência }(n=769)\end{array}$} & \multirow{3}{*}{$p^{*}$} & \multirow{3}{*}{$p^{* *}$} \\
\hline & \multicolumn{2}{|c|}{ Pouco ativo } & \multicolumn{2}{|c|}{ Ativo } & \multicolumn{2}{|c|}{ Pouco ativo } & \multicolumn{2}{|c|}{ Ativo } & & \\
\hline & $\mathbf{n}$ & $\%$ & n & $\%$ & $\mathbf{n}$ & $\%$ & $\mathbf{n}$ & $\%$ & & \\
\hline \multicolumn{11}{|l|}{ Domínios } \\
\hline Total & 113 & 38,6 & 180 & 61,4 & 305 & 39,7 & 464 & 60,3 & 0,92 & 0,86 \\
\hline Trabalho & 278 & 94,9 & 15 & 5,1 & 720 & 93,6 & 49 & 6,4 & 0,53 & 0,66 \\
\hline Transporte & 261 & 89,1 & 32 & 10,9 & 613 & 79,7 & 156 & 20,3 & 0,001 & 0,32 \\
\hline Atividades Domésticas & 183 & 62,5 & 110 & 37,5 & 620 & 80,6 & 149 & 9,4 & $<0,001$ & $<0,001$ \\
\hline Lazer & 199 & 67,9 & 94 & 32,1 & 576 & 74,9 & 193 & 25,1 & 0,06 & 0,26 \\
\hline
\end{tabular}

* comparação da proporção de pouco ativos entre participantes e não participantes de grupos de convivência. ** comparação da proporção de ativos entre participantes e não participantes de grupos de convivência.

damental), que pode estar relacionada à baixa renda, pois a participação nos grupos de convivência é uma opção de lazer economicamente favorável para aqueles que recebem baixas aposentadorias e/ou pensões ${ }^{19}$, realidade essa que é comum em diferentes regiões do Brasil. Nesse contexto, esses grupos passam a ser uma importante alternativa de convívio social para essa população, principalmente para as mulheres, que representam a maioria expressiva nesses grupos ${ }^{11,18}$.

Quanto à relação das condições de saúde e participação em grupos de convivência foram observadas associações estatísticas significantes, ou seja, as idosas do GP relataram ter mais doenças, porém têm percepção positiva do estado de saúde. Em concordância com esse achado, investigação realizada em Minas Gerais detectou melhor estado geral de saúde para os idosos participantes de grupos de convivência quando comparado com os que não participavam ${ }^{20}$.

Destacando a autopercepção de saúde, de forma geral, os idosos autoavaliaram a saúde como boa, sendo a maior prevalência para o GP $(83,8 \%$ e $74,1 \%$ para homens e mulheres, respectivamente). Esses resultados podem ser considerados superiores aos encontrados na investigação rea- 
Tabela 3. Associação entre as condições de saúde e nível de atividade física em idosos participantes ou não de grupos de convivência, segundo o sexo. Florianópolis, Santa Catarina, 2002.

\begin{tabular}{|c|c|c|c|c|c|c|c|}
\hline \multirow[b]{3}{*}{ Variáveis } & \multicolumn{6}{|c|}{ Grupos de Convivência } & \multirow[b]{3}{*}{$p$} \\
\hline & \multicolumn{2}{|c|}{ Participantes } & \multicolumn{2}{|c|}{ Não participantes } & \multicolumn{2}{|c|}{ Total } & \\
\hline & $\mathbf{n}$ & $\%$ & $\mathbf{n}$ & $\%$ & $\mathbf{n}$ & $\%$ & \\
\hline \multicolumn{8}{|l|}{ Mulheres } \\
\hline Presença de doença & & & & & & & $0,01^{\star}$ \\
\hline Sim & 216 & 81,2 & 260 & 72,4 & 476 & 76,2 & \\
\hline Não & 50 & 18,8 & 99 & 27,6 & 149 & 23,8 & \\
\hline Percepção do estado de saúde & & & & & & & $<0,001^{*}$ \\
\hline Boa & 223 & 83,8 & 247 & 68,8 & 470 & 75,2 & \\
\hline Ruim & 43 & 16,2 & 112 & 31,2 & 155 & 24,8 & \\
\hline Nível de Atividade Física Total & & & & & & & 0,57 \\
\hline Ativo & 163 & 61,3 & 212 & 59,1 & 375 & 60,0 & \\
\hline Pouco ativo & 103 & 38,7 & 147 & 40,9 & 250 & 40,0 & \\
\hline \multicolumn{8}{|l|}{ Homens } \\
\hline Presença de doença & & & & & & & 0,94 \\
\hline Sim & 19 & 70,4 & 291 & 71,0 & 310 & 70,9 & \\
\hline Não & 08 & 29,6 & 119 & 29,0 & 127 & 29,1 & \\
\hline Percepção do estado de saúde & & & & & & & 0,67 \\
\hline Boa & 20 & 74,1 & 288 & 70,2 & 308 & 70,5 & \\
\hline Ruim & 07 & 25,9 & 122 & 29,8 & 129 & 29,5 & \\
\hline Nível de Atividade Física Total & & & & & & & 0,87 \\
\hline Ativo & 17 & 63,0 & 252 & 61,5 & 269 & 61,6 & \\
\hline Pouco ativo & 10 & 37,0 & 158 & 38,5 & 168 & 38,4 & \\
\hline
\end{tabular}

*diferença estatística significante pelo Teste Qui-quadrado $(\mathrm{p}<0,05)$.

lizada com amostra representativa da população idosa brasileira, que foi $43,6 \%{ }^{19}$ e no estudo de Borges e col..$^{18}$ que 51,2\% autoperceberam a saúde como boa.

Em relação aos níveis de atividade física total, a maioria dos idosos foi classificada como ativa fisicamente. Todavia, quando realizada a análise pelos domínios do IPAQ, a maioria dos idosos do GP e do GNP foram considerados pouco ativos em todos os domínios. Ressalta-se que a influência do ambiente praiano, diversas ações de atenção à saúde junto à população idosa de Florianópolis, como os projetos de atividades física da Secretaria de Ação Social, das universidades, do SESC, entre outros, podem influenciar a manutenção ou a melhora do nível de atividade física dos idosos tanto do GP quanto do GNP, em especial, para o domínio das atividades de lazer.

Dentre estas atividades, destaca-se que a prática de exercício físico regular melhora o nível de atividade física dos idosos e contribui para manutenção e/ou melhoria da capacidade funcional e, consequentemente, contribui para o envelhecimento saudável ${ }^{8,10,11}$. Porém, faz-se necessário investir em estratégias para o aumento dos níveis de atividade física dos idosos, sejam eles participantes ou não de grupos de convivência. Além dos contextos destacados como os que apresentaram maiores prevalências entre os idosos ativos do GP (atividades domésticas e de lazer) e do GNP (atividade de lazer e transporte), outras ações poderiam auxiliar o aumento desses níveis. Dentre essas ações, destaca-se o estímulo às atividades de voluntariado após a aposentadoria, para o contexto do trabalho. E para o transporte, ações de políticas públicas (melhora das calçadas, da iluminação, da segurança, dentre outros) e de conscientização dos benefícios do deslocamento ativo para a saúde.

Quanto ao nível de atividade física e a participação em grupos de convivência não foi observada associação estatística significante. Diferentemente desse achado, pesquisa de Mazo e col. ${ }^{10}$ realizada com idosas participantes de centros de convivência evidenciou associação entre os níveis de atividade física (ativo e pouco ativo).

Considerando o aumento da população idosa, deve-se ampliar a rede de atendimento comunitário aos idosos com atuação mais efetiva de outras instituições/entidades, bem como implan- 
tar outras modalidades de atendimento e atividades de acordo com o interesse e a necessidade dos mesmos, sensibilizando a maior participação. Faz-se oportuno, ainda, refletir sobre o processo de inclusão da população idosa nos grupos de convivência de Florianópolis, em especial para os homens. Além disso, deve-se considerar que nem todos têm acesso aos grupos de convivência e programas de atividades físicas ofertados, seja por desconhecimento, desinteresse, falta de condições ou por ter outros compromissos.

Portanto, os grupos de convivência de Florianópolis devem repensar e propor atividades alternativas para atrair aqueles que ainda são minoria nos grupos, como homens idosos, pessoas com percepção negativa do estado de saúde, dentre outros. Além disso, faz-se necessário criar programas, incentivar e esclarecer os benefícios da participação nos mesmos, por meio da ampla divulgação à população.

\section{Conclusão}

Os resultados encontrados evidenciaram associação entre as condições de saúde (percepção do estado de saúde e presença de doença) e participação em grupos de convivência. Porém, a associação foi significante somente para o sexo feminino. O nível de atividade física não mostrou-se associado. A maioria dos idosos foi considerada ativa (nível de atividade física total). Os idosos engajados em grupos de convivência foram mais ativos que os não participantes, e as mulheres participam mais dos centros de convivência para idosos. Observou-se, ainda que, as doenças acometem a maioria dos idosos, e em maior percentual as mulheres participantes de grupos de con- vivência. Porém, tanto os homens quanto as mulheres participantes desses grupos têm percepção positiva do seu estado de saúde em relação aos que não participam.

Sugere-se a realização de outras investigações abordando as variáveis utilizadas para futuras comparações e análises. Estudos longitudinais poderão ser interessantes para a compreensão da relação entre condições de saúde e nível de atividade física em idosos participantes e não participantes de grupos de convivência.

\section{Colaboradores}

TRB Benedetti e GZ Mazo participaram da concepção e do delineamento do estudo; redação do artigo e aprovação da versão a ser publicada. LJ Borges participou da análise e da interpretação dos dados, redação do artigo e aprovação da versão a ser publicada.

\section{Agradecimentos}

Ao Ministério de Saúde e CNPq pelo financiamento das pesquisas. 


\section{Referências}

1. Organização Pan Americana da Saúde (OPAS). Envelhecimento: mitos na berlinda. Brasília: OPAS; 1999.

2. Brasil. Lei no 8.842 de 4 de janeiro de 1994. Dispõe sobre a política nacional do idoso cria o Conselho Nacional do Idoso e dá outras providências. Diário Oficial da União 1994; 5 jan.

3. Brasil. Lei $n^{\circ} 10.741$, de 01 de outubro de 2003. Dispõe sobre o Estatuto do Idoso e dá outras providências. Diário Oficial da União 2003; 3 out.

4. Instituto Brasileiro de Geografia e Estatística (IBGE). Pesquisa Nacional por Amostra de Domicílio, 2006. Síntese dos indicadores sociais 2007. v. 21. Rio de Janeiro: IBGE; 2007.

5. Diogo MJE, Duarte YAO. Cuidados em domicílio: conceitos e práticas. In: Freitas EV, Py L, organizadores. Tratado de geriatria e gerontologia. Rio de Janeiro: Editora Guanabara Koogan; 2002. p. 762-767.

6. Pitkala KH, Blomquist L, Routasalo P, Saarenheimo M, Karvinen E, Oikarinen U, Mantyranta T. Leading groups of older people: a description and evaluation of the education of professionals. Educ Gerontol 2004; 30(10):821-833.

7. Miguel CS, Fortes VLF. Idosas de um grupo de terceira idade: as interfaces da relação com suas famílias. RBCEH 2005; 2(2):74-85.

8. American College of Sports Medicine position stand, Chodzko-Zajko WJ, Proctor DN, Fiatarone Singh MA, Minson CT, Nigg CR, Salem GJ. Skinner JS. Exercise and Physical Activity for Older Adults. Med Sci Sports Exerc 2009; 41(7):1510-1530.

9. Mazo GZ, Mota J, Gonçalves LHT, Matos MG, Carvalho J. Actividade física e qualidade de vida de mulheres idosas da cidade de Florianópolis, Brasil. Rev Port Cien Desp 2008; 8(3):414-423.

10. Mazo GZ, Mota J, Gonçalves LHT, Matos MG. Nível de atividade física, condições de saúde e características sócio-demográficas de mulheres idosas brasileiras. Rev Port Cien Desp 2005; 5(2):202-212.

11. Cardoso AS, Levandoski G, Mazo GZ, Prado APM, Cardoso LS. Comparação do nível de atividade física em relação ao gênero de idosos participantes de grupos de convivência. RBCEH 2008; 5(1):9-18.

12. Toscano JJO, Oliveira ACC. Qualidade de Vida em Idosos com Distintos Níveis de Atividade Física. Rev Bras Med Esporte 2009; 15(3):169-173.

13. Instituto Brasileiro de Geografia e Estatística (IBGE). Sintese de Indicadores Social, 2000. Rio de Janeiro: IBGE; 2000.
14. Mazo GZ. Atividade Física e Qualidade de Vida de Mulheres Idosas [tese]. Porto, Portugal: Faculdade de Ciências do Desporto e de Educação Física; 2003.

15. Benedetti TRB. Atividade Física: uma perspectiva de promoção da saúde do idoso no município de Florianópolis [tese]. Florianópolis: Universidade Federal de Santa Catarina; 2004.

16. Veras RP, Dutra S. Questionário BOAS (Brazil Old Age Schedule) versão 2000. [página na Internet]. [acessado 2001 out 23]. Disponível em: http:// www.unati.uerj.br/

17. Nelson ME, Rejeski WJ, Blair SN, Duncan PW, Judge JO, King AC, Macera CA, Castaneda-Sceppa C. Physical activity and public health in older adults: recommendation from the American College of Sports Medicine and the American Heart Association. Circulation 2007; 116(9):1094-1105.

18. Borges PLC, Bretãs RP, Azevedo SF, Barbosa JMM. Perfil dos idosos frequentadores de grupos de convivência em Belo Horizonte, Minas Gerais, Brasil. Cad Saude Publica 2008; 24(12):2798-2808.

19. Mazo GZ, Lopes MA, Benedetti TB. Atividade física e o idoso: Concepção Gerontológica. 3a edição. Porto Alegre: Editora Sulina; 2009.

20. Almeida EA, Madeira GD, Arantes PMM, Alencar MA. Comparação da qualidade de vida entre idosos que participam e idosos que não participam de grupos de convivência na cidade de Itabira-MG. Rev. Bras. Geriatr. Gerontol. 2010; 13(3):435-444.

Artigo apresentado em 15/05/2011

Aprovado em 04/09/2011

Versão final apresentada em 26/09/2011 